\title{
Multistrand, Fast Reaction, Shape Memory Alloy System for Uninhabited Aerial Vehicle Flight Control
}

\author{
M. Brennison, R. M. Barrett, and L. Kerth \\ Department of Aerospace Engineering, University of Kansas, 2120 Learned Hall, 1530 W. 15th St. Lawrence, KS 66045, USA \\ Correspondence should be addressed to R. M. Barrett, barrettr@ku.edu
}

Received 15 November 2011; Accepted 14 June 2012

Academic Editor: Marcelo A. Trindade

Copyright () 2012 M. Brennison et al. This is an open access article distributed under the Creative Commons Attribution License, which permits unrestricted use, distribution, and reproduction in any medium, provided the original work is properly cited.

This paper details an investigation of shape memory alloy (SMA) filaments which are used to drive a flight control system with precision control in a real flight environment. An antagonistic SMA actuator was developed with an integrated demodulator circuit from a JR NES 911 subscale UAV actuator. Most SMA actuator studies concentrate on modeling the open-loop characteristics of such a system with full actuator performance modeling. This paper is a bit different in that it is very practically oriented and centered on development of a flight-capable system which solves the most tricky, practical problems associated with using SMA filaments for aircraft flight control. By using well-tuned feedback loops, it is shown that intermediate SMA performance prediction is not appropriate for flight control system (FCS) design. Rather, capturing the peak behavior is far more important, along with appropriate feedback loop design. To prove the system, an SMA actuator was designed and installed in the fuselage of a $2 \mathrm{~m}$ uninhabited aerial vehicle (UAV) and used to control the rudder through slips and coordinated turns. The actuator was capable of 20 degrees of positive and negative deflection and was capable of $7.5 \mathrm{in}-\mathrm{oz}(5.29 \mathrm{~N} \mathrm{~cm})$ of torque at a bandwidth of $2.8 \mathrm{~Hz}$.

\section{Introduction}

1.1. Background. Irreversible aircraft flight control is typically achieved by using some form of conventional hydraulic or electromechanical servoactuators. These types of actuators are often quite large, heavy, and use numerous moving parts. For more than 20 years, adaptive structures technologists have worked to reduce weight, volume, and power consumption of these actuators by employing adaptive materials. The earliest inklings of flight control via adaptive aerostructures were seen in the 1980s with experiments on various kinds of twisting and bending plates [1-3]. These early experiments were followed by a slew of innovations directed towards control of rotorcraft via actively twisting structures, flaps, and pitch activation mechanisms [4]. A host of missile and munition applications first came around in the 1990s employing adaptive aerostructures in pitch-, camber-, and twist-active fins, canards, and wings [5-9]. Helicopter rotors were manipulated in their entirety and flown for the first time in 1997 [10]. These adaptive rotors were morphed into the DoD's first successful Micro Aerial Vehicles with a program starting in 1994. This early effort eventually lead to $6^{\prime \prime}$ internal combustion-engine powered, adaptive structures controlled rotorcraft successfully flying off in a DARPA competition at Quantico, VA, in 1990 [11]. Following that flyoff, a series of ultrahigh performance convertible UAVs were made using adaptive structures. One of the major innovations used in this singular class of UAVs was the new Post-Buckled Piezoelectric (PBP) flight control actuators. Because the aircraft employed grid fins, it possessed a very high empennage normal force gradient with minimal aerodynamic pitching moments and no stall break. Of course, the high gradients meant that only comparatively small deflections were actually needed for full flight control through $90^{\circ}$ deck angle changes.

Although piezoelectric actuators have clearly been shown to be quite applicable to the task of ultra-high performance UAV flight control, several classes of UAVs will not need such extreme control forces as they only perform simple maneuvers as drag minimization is an optimization factor for maintenance of high $\mathrm{L} / \mathrm{D}$. Towards that end, conventional flight control surfaces and stabilizers still have a necessary place in the arsenal of techniques available to the aircraft design engineer. For UAVs with a human controller in 


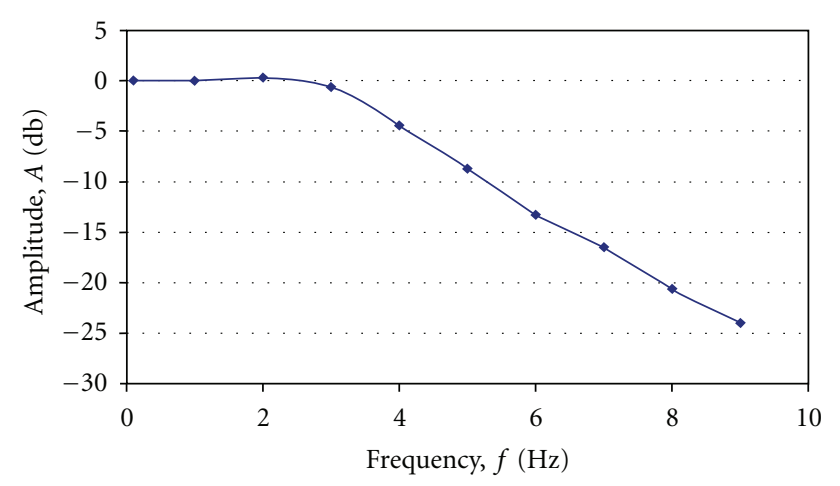

FIGURE 1: Bode amplitude plot of a cirrus CS-10BB sub-submicro servoactuator with corner frequency of approximately $3 \mathrm{~Hz}$.

the loop, it can be seen that typically the highest frequency flight dynamics mode expected to be covered is the short period which typically ranges from $0.5-3.5 \mathrm{~Hz}$ for $1 \mathrm{~m}-3 \mathrm{~m}$ size fixed-wing aircraft.

If one examines the pantheon of adaptive materials available to the aircraft flight control system design engineer, it can be seen that shape memory alloys (SMAs) fit this actuator bandwidth norms quite well. Figure 1 shows the Bode amplitude plot with associated roll-off at approximately $3 \mathrm{~Hz}$ of a common sub-submicro servoactuator like that used in aircraft of this category.

Although piezoelectric actuators have been shown to work and work well, they almost always require comparatively expensive high voltage electronics to function properly. Because SMA actuators sized for a $2 \mathrm{~m}$ aircraft would operate on voltages which are compatible with COTS electronics, it is easy to see that the total cost of a SMA-controlled UAV could be quite competitive. Because the cost of the SMA filaments with respect to electromechanical actuator cores is also competitive, there exists a window of opportunity to demonstrate a highly useful form of flight control with SMA filaments, this study will examine its utility as an actuator replacing a single servoactuator on a $2 \mathrm{~m}$ sized UAV.

\section{General Description}

2.1. SMA Properties. SMAs are fairly unique among actuator materials because they have the ability to return to a trained shape, achieved by changes in the alloy's atomic crystal structure when heat is applied. An SMA has two primary states of atomic structure: martensitic and austenitic. When an SMA is in the martensitic state, it is at room temperature and relaxed. Once heat is added to an SMA, the atomic structure changes and is between the martensitic and austenitic state. If enough heat is added so that the SMA just exceeds the austenitic phase transformation temperature, it becomes fully austenitic. Given a plethora of references on these properties, the studies in [12-14] offer good primers on the subject.

SMAs are actuated when a source of heat applied. In this study, when heat was applied to an SMA line, it would contract in length. The preferred method of providing heat to SMA's for aircraft applications is by electrical resistance. The
SMA lines that were used in this study were $.003^{\prime \prime}(12.7 \mu \mathrm{m})$ in diameter. The $.003^{\prime \prime}$ SMA's were capable of pulling a maximum force of 2.82 ounces and provided $5 \mathrm{Ohms}$ of resistance for every inch of length of the SMA line. When an electric current flows through the SMA, the resistance of the line against the current creates friction which in turn generates enough heat to actuate the SMA.

In order for the SMA lines to receive electricity, electrical contacts are required to remain at fixed points on the SMA line. Alligator clips could slide along the SMA line which can cause only a portion of the desired amount of the SMA to be actuated. Soldering electrical wires to fixed points along the SMA would be ideal; however, solder would not adhere to the SMA due to extremely high active strains. To circumvent the soldering problem, $1 / 8^{\prime \prime}(3.18 \mathrm{~mm})$ lengths of $1 / 32^{\prime \prime}(0.79 \mathrm{~mm})$ ID brass tubing were crimped at opposite ends of the SMA line. Electrical wires were then soldered to the brass tubing so that electricity could be provided to the SMA. Alternatively, the SMA line can be wound around the threads to steel "hollow-bolts," and the electrical wires may be soldered to the bolts. When the SMA was wrapped around steel bolts, there was a possibility that the strain of the SMA would relax and the SMA could off the bolt, opening the circuit. To prevent the SMA from detaching from the steel bolt, aluminum tape was used to keep the SMA on the bolt and allow the SMA to slide along the bolt threads. Both methods of applying electrical contacts are shown in Figure 2.

2.2. Actuator Systems. It is possible to design an SMA actuator that had similar characteristics of mechanical and hydraulic actuators used for flight control systems. To produce a deflection of an SMA actuator was as simple as adding an appropriate level of electric current to one actuator, but to return from the deflection required a force to oppose the SMA. Prior research at Auburn University had developed two primary systems of providing an opposing force: the Pull-Spring System and the Antagonistic Pull-Pull System. In both systems, the SMA's were preheated beyond the Austenitic finish using a heat gun before they were strained.

The Pull-Spring System used a single SMA line, a spring, and a lever that pivots about its center. The SMA is attached to a fixed point and extends to one end of the lever. A spring was attached to the other end of the lever and was stretched to another fixed point. An illustration of the Pull-Spring System is shown in Figure 3.

The spring served a dual purpose as it provided a force that opposed the SMA as well as maintained a strain in the SMA at all times. If the SMA was in the martensitic state, the spring would contract, causing a clockwise deflection of the lever, straining the SMA. To keep the system in the neutral position, enough heat must be added to the SMA so that the pull force of the SMA equaled the force of the spring. To cause a counterclockwise deflection, even more heat must be added to the SMA so that its pull strength was greater than the force of the spring. Because the linear deflections of the spring were small during operation of the system, the spring force was considered constant. 


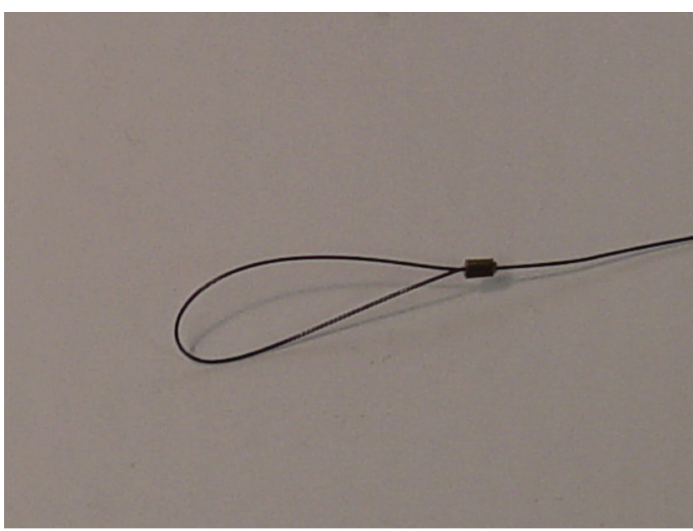

(a)

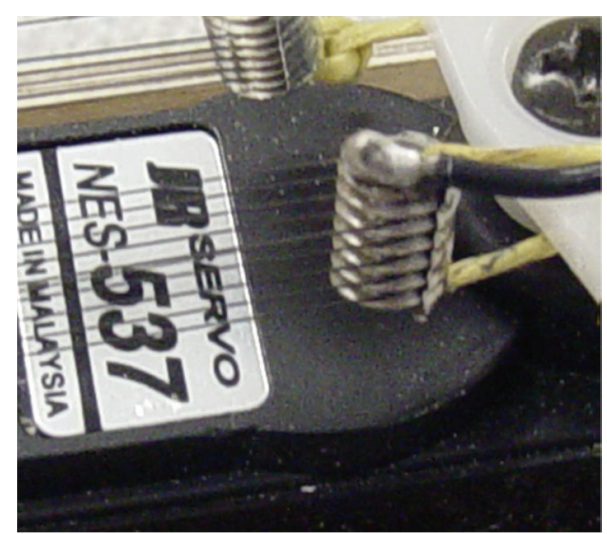

(b)

FIGURE 2: Electrical contact implementations: crimped brass tubing (a) and steel bolt threads.

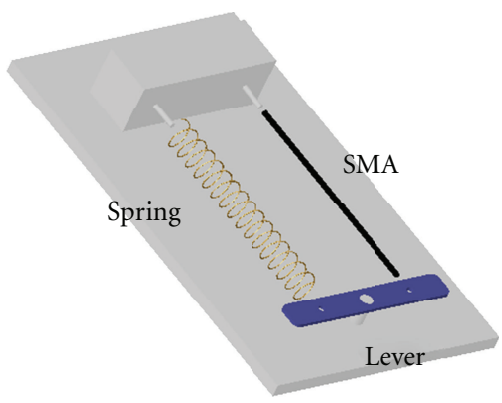

Figure 3: Pull-Spring system [5].

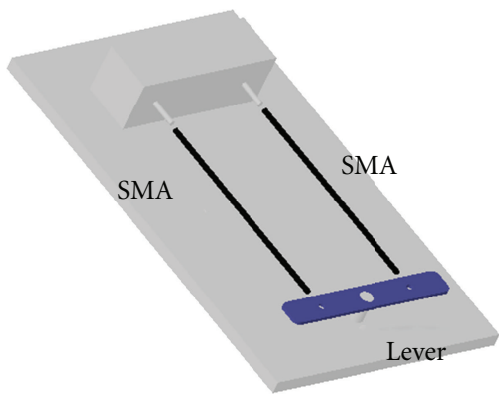

Figure 4: Antagonistic system [5].

The advantage to using the Pull-Spring System was that only a single SMA line had to be controlled. By using a single SMA line, the electrical system was tremendously simplified. The disadvantage of the Pull-Spring System was that the SMA was almost constantly fighting the force of the spring, causing a greater than desired amount of power consumption.

An Antagonistic System was similar to the Pull-Spring System with the exception that the spring was replaced by a first SMA line. An illustration of the Antagonistic System is shown in Figure 4.

The lever was placed in the neutral position and then both SMA lines were stretched to achieve a prestrain of 3\% in each line. Both SMA lines were in the martensitic state while the lever was in the neutral position. To deflect the lever, one of the SMA lines must be heated. As an SMA line departed the martensitic state, it contracted, which turned the lever and strained the opposing SMA line. Once the heat source was removed, the contracted SMA line would relax and allow the actuator to move slightly toward the neutral position due to strain relief of the opposing SMA line. To return to the neutral position, an electrical current was applied to the strained SMA line, which caused it to contract.

There were two main advantages from using the Antagonistic system. Firstly, the Antagonistic System was more efficient since no power was required when the system was in the neutral position. Additionally, the Antagonistic System operated more like a conventional mechanical actuator than the Pull-Spring System. For these two reasons, it was determined that the Antagonistic system was better suited for the study.

When both of the SMA lines were strained, the stress level of the actuator increased. However, as the stress level was increased, the amount an SMA line could actively contract, as well as the amount of deflection of the actuator, decreased. Figure 4 shows two stress-strain graphs plotted against each other, for an Antagonistic system. At the points of intersection of the two graphs, the actuator was in the neutral position. The lowest pair curves represent the martensitic phase and highest pair of curves represents the austenitic phase. As the temperature of both SMA lines in the martensitic phase increased, the stress level also rose. By increasing the stress level, the system became more ridged against outside forces. The downside of increasing the stress level was the amount of deflection that the actuator was capable of was reduced. This phenomenon is illustrated in Figure 5.

2.3. Traditional Servoactuator. Traditionally with radio controlled unmanned aerial vehicles (UAVs), an operator on the ground sends command inputs to the aircraft, via radio waves, in a sequence of electrical pulses, over a discontinuous sine wave, using an oscillating-crystal radio transmitter. Onboard aircraft, a crystal-controlled receiver takes the signals from the transmitter. Pulse-width modulated signals 


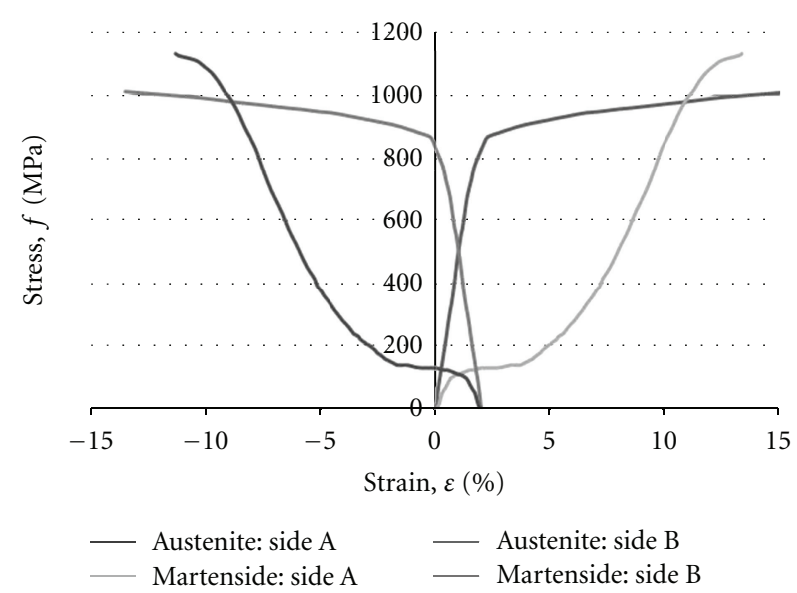

FIGURE 5: Stress versus strain graph of an antagonistic system [7].

from the receiver are sent to a demodulator circuit within the servo. The demodulator circuit takes the signal and outputs a corresponding voltage supplied by a battery. The output voltage causes a motor within the servo to rotate which in turn causes a set of gears and the control arm, attached a potentiometer, to rotate as well. The polarity of the voltage output by the demodulator circuit will determine whether the motor shaft will rotate clockwise or counterclockwise. These components will continue to rotate until the resistance of the potentiometer is enough to balance the system, meaning the voltage output of the system is zero and the motor will stop rotating. If the system was held at the same deflection but an external force, such as an aerodynamic load, was imparted on the system such that the deflection would change, the balance is destroyed and more power was supplied to the system so that it can remain balanced and at the same position; thus, closed loop electromechanical feedback was achieved.

2.4. Development of the SMA Actuator. The design of the SMA actuator would follow similar philosophies in the design of traditional servoactuators. Utilizing an existing demodulator circuit, as well as a potentiometer as a means of detecting the amplitude of deflection, a feedback loop was established; this worked well with a linear system and was believed that even with the nonlinear behavior of SMA, the power could be continually increased until the desired amount of deflection by the SMA was achieved. To test the concept, a simple breadboard apparatus was constructed that used two SMA wires in an antagonistic system. For this experiment, the demodulator circuit, potentiometer, and plastic housing of a JR NES 911 servo were salvaged; the demodulator circuit would operate normally as though it was connected to servo components and the plastic housing was used as a convenient platform with rotational assembly to which the SMA could be attached. The potentiometer from the servo remained attached to the control arm as well as to the demodulator circuit for position feedback. The power output from the demodulator circuit that would normally be used to drive the servo motor would be used to supply power to the two SMA wires that would drive the actuator. The

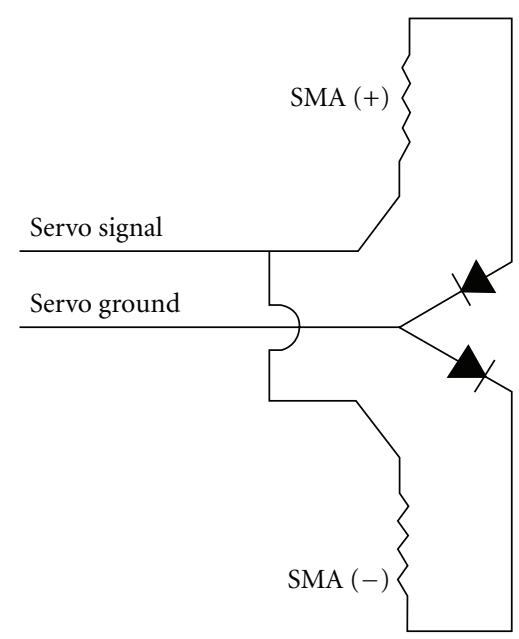

FIGURE 6: Electrical schematic of first prototype.

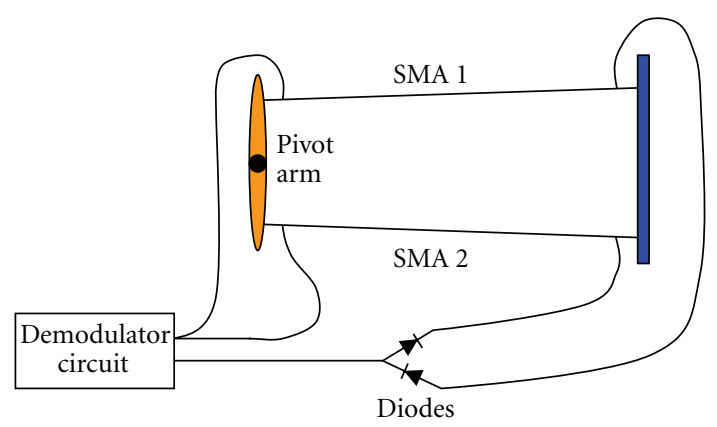

FIgURE 7: Mechanical diagram of first prototype.

voltage output from the demodulator circuit would be either negative or positive polarity; however, SMA is indiscriminate of voltage polarity. To supply power to only one SMA wire at a time, a pair of opposing diodes was attached to the demodulator circuit ground output as well as to the SMA wires as illustrated in Figures 6 and 7.

Each SMA wire was connected to one side of the control arm as well as a prestrain mechanism that was used to initially strain each SMA wire by $3 \%$. Brass electrical connections were applied at the ends of each SMA wire to supply power along the entire length of the wire. In place of a transmitter and receiver, a servociser was used to generate pulse-width modulated (PWM) signals sent to the demodulator circuit. The entire system was powered by a 6 cell, 7.2 v NiCad battery. The apparatus is shown in Figure 8 .

The experiment exhibited that as command inputs were sent to the demodulator circuit, there was a corresponding contraction of one of the SMA lines. The SMA contraction would cause the control arm and the potentiometer to turn until the potentiometer balanced the system. The experiment also proved the actuator could be commanded to different positions repeatedly.

The apparatus proved the concept was sound; however, it also revealed a major disadvantage. The SMA lines required a significant amount of power, about 1.35 watts, to make rapid, accurate deflections. To design an airworthy SMA 


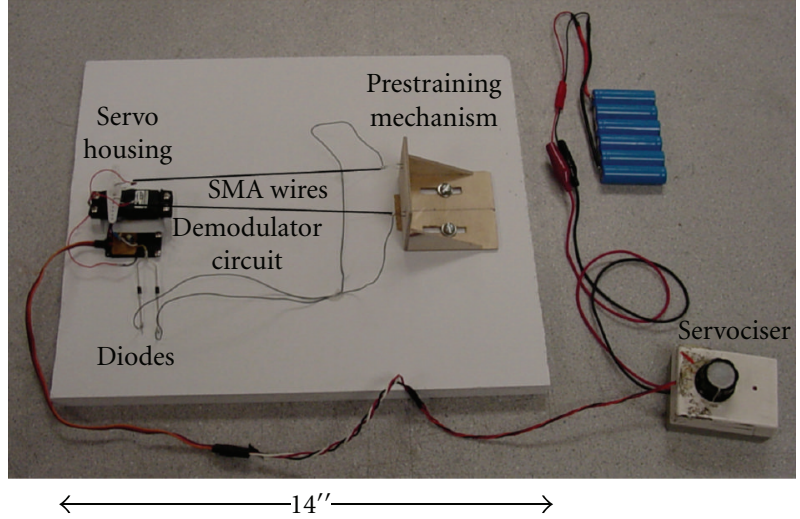

Figure 8: First prototype with diodes.

actuator, a significant increase in both length and number of stands of SMA wire would be required which corresponded to an increased power requirement. The SMA could handle the increase in power applied; however, the maximum power output of the demodulator circuit was 1.35 watts. Supplemental power would need to be provided to the SMA to sufficiently actuate the wire and the amount of additional power supplied to the SMA needed to be proportional to the power output from the demodulator circuit. It was also necessary to ensure that the supplemental power did not flow into the demodulator circuit; failure to prevent this would result in the destruction of the demodulator circuit.

2.5. Transistors. The breadboard apparatus was modified such that each SMA wire was provided with a supplemental power supply and each diode was replaced with a bipolar transistor. Both of the transistors have three leads: base, collector, and emitter. The base is a gate that closes the circuit of a large power supply, connected to the collector and emitter, when triggered by a small power supply. A power supply provided 4.8 volts to the demodulator circuit and based upon the user input, produced a voltage sent through the signal lead to the base of one of the two transistors, depending on the polarity of the current. The current that flows through the base closes the gate and allows a supplemental 7.2 volt battery to activate the corresponding SMA line. As the signal voltage decreased, the gate opened the circuit of the supplemental power supply. As the signal voltage was altered, the transistor opened and closed the gate instantaneously. The instantaneous changes by the transistor was important because it was much more energy efficient than making a gradual change. The transistors worked in a similar fashion to the diodes: the base of the PNP transistor would only connect the collector and the emitter when positive polarity was applied and on NPN when negative polarity was applied to the base. The NPN and PNP transistors have created a system that will allow only one SMA line to be active at a time.

A schematic of the breadboard with transistors is shown in Figure 9. Figure 10 shows a conceptual layout of the power supply-to-demodulator-to-electronics-to-SMA mechanical effectors arrangement.

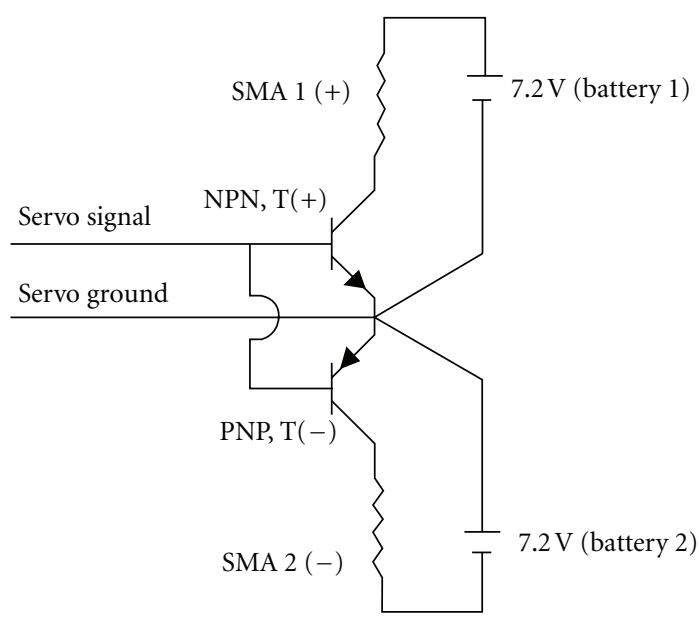

FIGURE 9: Electrical schematic of second prototype.

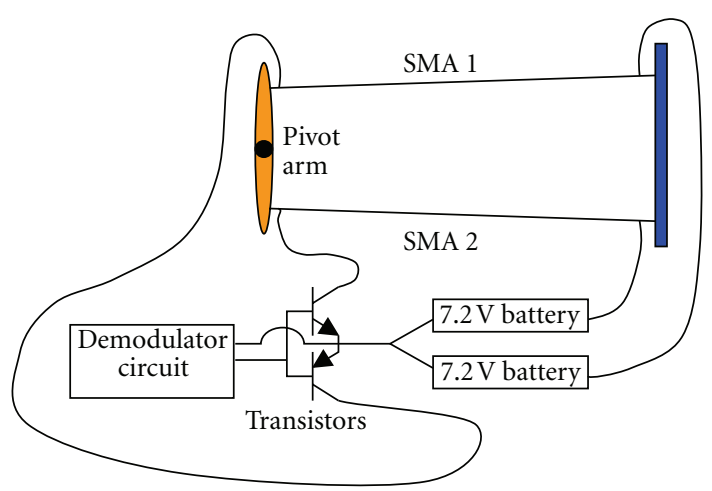

FIGURE 10: Mechanical diagram of second prototype.

Just as with the diode breadboard, the SMA lines were initially preheated and prestrained $3 \%$ by stretching them with a prestrain mechanism. The second prototype is shown in Figure 11.

The apparatus proved it could precisely control position control of the actuator as well as meet the power requirements without damaging the demodulator circuit; thus, this design was suitable for flight.

The flight test article was assembled on a plywood module so that it could easily be installed and removed from the aircraft without damaging the actuator. The modular design also allowed the actuator to be tested prior to being installed in the UAV without dismantling the actuator.

The module had a slightly different design than the breadboard apparatus. The laboratory prototype only had a single strand pulling both directions; however, the module actuator had ten. The module used a single 0.003 in diameter SMA line, for each direction of pull, that was wrapped five times around two threaded bolts, achieving the same strength as though ten separate SMA lines were used. By using this configuration, the actuator was able to produce $7.5 \mathrm{oz}^{*}$ in of torque, the same amount possible by a similarly sized servo. To achieve a desired deflection of 20 degrees, the SMA "strands" needed to be $4.75 \mathrm{in}(12.07 \mathrm{~cm})$ long; when the strands contract as much as $3 \%$ in conjunction with 


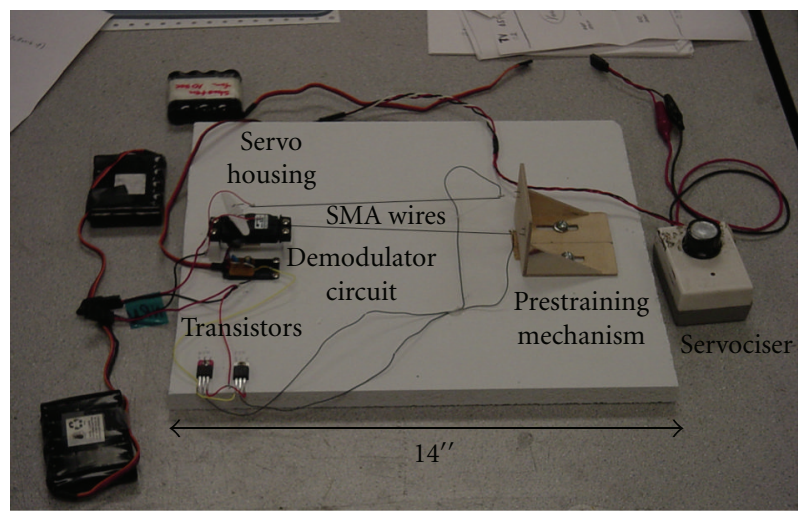

FIGURE 11: Second prototype with power transistors.

being spaced 0.188 in $(4.76 \mathrm{~mm})$ from the control arm hub, the desired angular deflection was achieved. The prestrain mechanism was covered in Teflon tape and anchored by a single nylon bolt and hex nut. The actuator module is shown in Figure 12.

When the module was installed in the UAV, it was held in place by a singly nylon bolt and hex nut as well as by the geometry of the inner walls of the fuselage. The two batteries that were used to actuate the SMA lines were stored in the UAV, forward of the actuator. To control the rudder, a linkage that extended to the rudder was connected to the control arm of the actuator.

2.6. Test Aircraft. To determine if the position control system had potential for flight applications, it would need to be flown and tested. The aircraft used for the test was a UAV that possessed the dimensions mentioned in Table 1.

For the test, it was determined that the SMA actuator should operate the rudder. If the SMA actuator were to fail in flight, the control surface it operated would become useless and possibly flutter. If the rudder were to become uncontrollable, it would still be possible to maintain controlled flight and make a safe landing using the ailerons and elevator. Should either the elevator or the ailerons fail, the UAV would be less controllable and pose more of a threat to individuals in the surrounding test area. Additionally, the rudder did not require as rapid or as many deflections as either the aileron or the rudder. Therefore, the rudder was deemed the safest control surface to use for testing the SMA actuator.

Major modifications were made to the aircraft, including the addition of a Norvel 074 gas-powered engine. The engine was added to increase airspeed and flight duration. The UAV would be flown at a field that was 200 yards $\times 75$ yards $(182.9 \mathrm{~m} \times 68.7 \mathrm{~m})$. During the flight tests, the UAV made several straight passes along the flight field with various rudder control inputs.

\section{Test Procedures}

Prior to operation of the actuator, the SMA lines were first preheated with a heat gun to return the SMA lines to their trained length. After the SMA lines were heated, they were
TABLE 1

\begin{tabular}{lcccc}
\hline & Wing & $\begin{array}{c}\text { Horizontal } \\
\text { tail }\end{array}$ & $\begin{array}{c}\text { Vertical } \\
\text { tail }\end{array}$ & \\
\hline Span & 66.5 & 22 & 6 & in \\
Area & 650 & 110 & 40.5 & in`2 $^{\wedge}$ \\
MGC LE FS & 12 & 38.5 & 33 & in \\
Aspect ratio & 6.8 & 4.4 & 0.9 & \\
Thickness ratio & 0.11 & & & \\
Total polyhedral angle & 8 & N/A & N/A & \\
Aileron chord ratio & 0.19 & N/A & N/A & \\
Aileron span ratio & 0.38 & N/A & N/A & \\
Root chord & 9.5 & 6.03 & 9.5 & in \\
Tip chord & 6 & 4.34 & 4.06 & in \\
Average chord & $\$ 7.75$ & \$5.18 & $\$ 6.78$ & in \\
\hline & Fuselage & & & \\
\hline Length & 47 & in & & \\
Maximum height & 1.91 & in & & \\
Minimum width & 2.17 & in & & \\
\hline
\end{tabular}

cooled and stretched 3\% of their original length. The SMA lines were prestrained by sliding a tray in the actuator to stretch the SMA and was then locked into place by a nylon bolt and hex nut tightened by a flathead screwdriver.

The actuator was then mounted in the UAV in a bay underneath the wings and anchored in place by a single nylon bolt and hex nut. After the actuator was in the correct position, the rudder control linkage was connected to the control arm of the actuator. The two 6 volt batteries, already installed in the aircraft, were then connected to the NPN and the PNP terminals of the actuator. Following the batteries, the signal line of the demodulator circuit was connected to the rudder channel of the receiver. All of connections to the batteries and to the receiver were double checked and then followed by the installation of the wing and forward bay canopy.

The transmitter was turned on prior to the UAV electronics to prevent the UAV from following any commands from erroneous source. The electronics aboard the UAV were then turned on supplying power to the receiver, servos, and the SMA actuator. To ensure proper communication between the transmitter and the receiver aboard the UAV, the transmitter sent commands to the receiver with the antennae at its minimum length from the opposite side of the flight field.

\section{Bench and Flight Test Results}

Laboratory test showed that the SMA actuator was capable of making deflections as great as 20 degrees in either direction and required less than $5 \mathrm{~V}$ for full deflection. During the flight test, it was observed from the ground that precise, controlled rudder deflections were possible by an SMA actuator. During the flight, several deflections were made to various positions besides maximum throw, proving that the position control system functions properly in flight. 


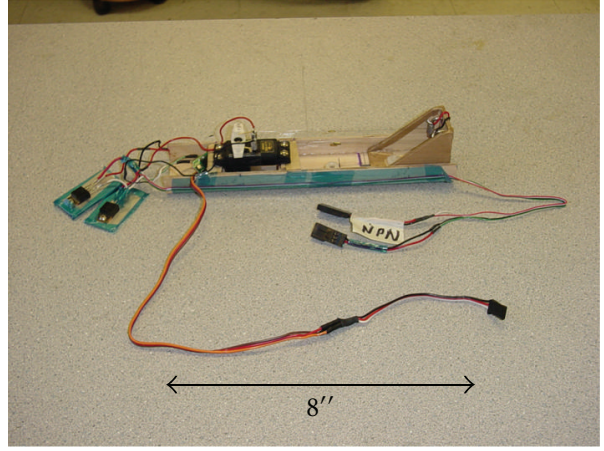

(a)

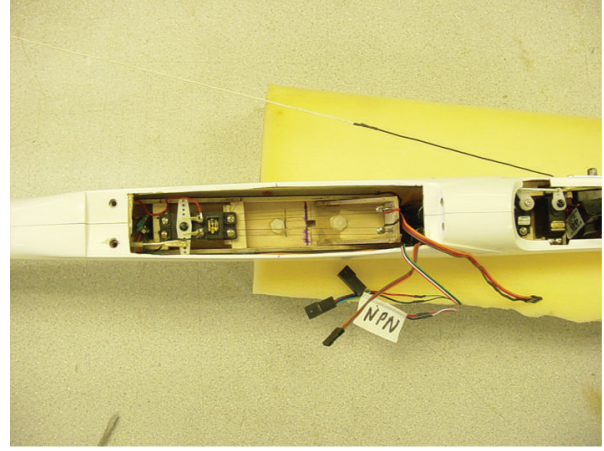

(b)

FIgURE 12: Flight-ready SMA actuator module (a), installed in UAV (b).

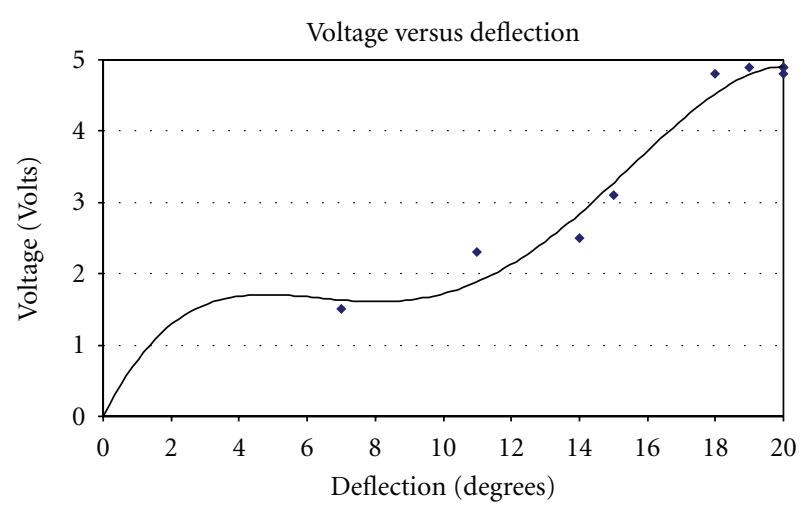

FIGURE 13: SMA voltage with deflection trends for actuator.

A parameter of interest was how much voltage was required for rudder deflection during flight. For this test, the actuator module was installed in the aircraft. Electrical wires were connected to the power lines at opposite ends of one of the SMA bundles to measure the voltage across the SMA with a voltmeter. At the tail, markings were placed so that deflection of the tail could be measured to the nearest degree. The aircraft was placed in an air stream and the actuator was commanded to turn the rudder to various deflection angles. At each deflection, the voltage and the magnitude of the deflection were recorded. The results of the test are presented in Figure 13.

The actuator was tested under a variety of conditions to determine environmental suitability. One of the more important tests was actuator phase lag under typical operating conditions. At $72 \mathrm{deg}$. F, with a cooling airstream of $20 \mathrm{ft} / \mathrm{s}, 60 \%$ relative humidity, the diode and transistor configurations of the actuator were tested. Rotational deflections were recorded by recording the measured resistance from the feedback potentiometer of the actuator system (measured accuracy under $0.02 \mathrm{deg}$. of rotation). Figure 14 shows the phase lag for a typical actuation signal with a period of $8 \mathrm{sec}$. over two complete cycles.

Once the engine was ready for flight, the UAV was hand launched while the engine was at full throttle. The

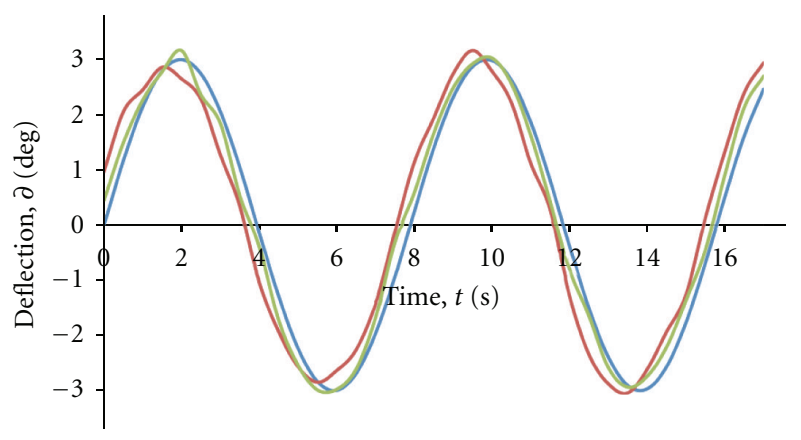

(a)

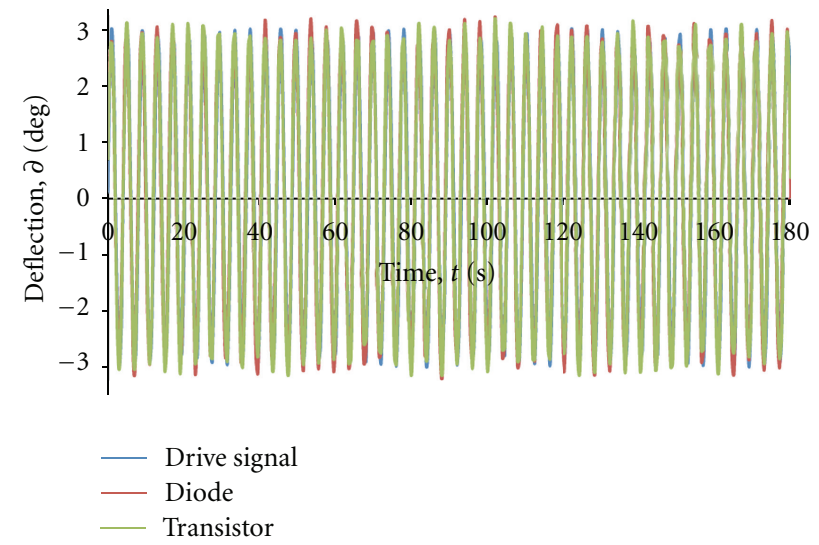

(b)

Figure 14: Phase lag given a sinusoidal driving signal with an $8 \mathrm{sec}$. period and repeatability of deflections over $3 \mathrm{~min}$. at $1 / 4 \mathrm{~Hz}$ of actuator driving signal, $72 \mathrm{deg}$. F, $20 \mathrm{ft} / \mathrm{s}$ airstream, $60 \%$ relative humidity for both the diode actuator system, and the power transistor actuator system.

UAV would then fly in a right traffic pattern to make sure everything was operating properly onboard. The UAV would remain in the traffic pattern and test the SMA actuator during a series of maneuvers performed on each pass along the flight field. Should the UAV not operate properly at any time during the flight, the test would be aborted and the UAV would land immediately. Figure 15 shows the UAV in a flight testing the SMA actuator. 


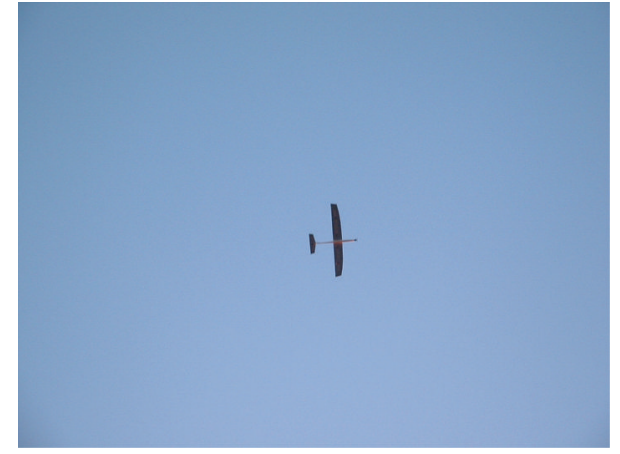

Figure 15: 2 m SMA controlled UAV in flight.

\section{Conclusions}

It can be concluded that SMA actuators were capable of producing precise control surface deflections for a UAV. Test results showed that full $\pm 20^{\circ}$ deflections could be generated by a $0.003^{\prime \prime}$ diameter SMA flight control system operating at voltages and current levels compatible with currents COTS radio-controlled electronics. It was also shown that power transistors coupled to said COTS electronics demonstrated superior performance over diode-equipped driving electronics providing control signals to the SMA flight control actuation systems.

\section{Acknowledgments}

The authors would also like to thank Dr. Christoph Burger for his piloting skills and so assisting with electronics design, fabrication, and testing. Additionally, the authors would like to thank the University of Kansas Transportation Research Institute (TRI) for its support for this work.

\section{References}

[1] E. Crawley, K. Lazarus, and D. Warkentin, "Embedded actuation and processing in intelligent materials," in Proceedings of the 2nd International Workshop on Composite Materials and Structures for Rotorcraft, Troy, NY, USA, September 1989.

[2] K. Lazarus and E. Crawley, "Multivariable active lifting surface control using strain actuation: analytical and experimental results," in Proceedings of the 2nd International Conference on Adaptive Structures, ASME, San Diego, Calif, USA, November 1992.

[3] K. B. Lazarus, E. F. Crawley, and J. D. Bohlmann, "Static aeroelastic control using strain actuated adaptive structures," in Proceedings of the 1st Joint U.S./Japan Conference on Adaptive Structures, Maui, Hawaii, USA, October 1990.

[4] R. L. Spangler and S. R. Hall, "Piezoelectric actuators for helicopter rotor control," in Proceedings of the 31st Structures, Structural Dynamics and Materials Conference, pp. 1589-1599, Long Beach, Calif, USA, April 1990.

[5] S. M. Ehlers and T. A. Weisshaar, "Static aeroelastic behavior of an adaptive laminated piezoelectric composite wing," in Proceedings of the 31st AIAA/ASME/ASCE/AHS/ASC Structures, Structural Dynamics and Materials Conference, pp. 1611-1623, April 1990.
[6] S. M. Ehlers and T. Weisshaar, "Adaptive wing flexural axis control," in Proceedings of the 2nd International Conference on Adaptive Structures, ASME, San Diego, Calif, USA, November 1992.

[7] S. M. Ehlers and T. A. Weisshaar, "Effect of material properties on static aeroelastic control," in Proceedings of the 33rd Structures, Structural Dynamics and Materials Conference, Dallas, Tex, USA, April 1992.

[8] G. Winchenbach, "Cone aerodynamics test," Aeroballistic Research Facility Ballistic Spark Range Technical Report, USAF Wright Laboratory Flight Vehicles Branch, WL/MNAV, 1996.

[9] G. Knowles, R. Barrett, and M. Valentino, "Self-contained high authority control of miniature flight control systems for area dominance," in Proceedings of the SPIE 11th International Symposium on Smart Structures and Materials, San Diego, Calif, USA, March 2004.

[10] G. Lee, "Design and testing of the kolibri vertical take-off and landing micro aerial vehicle," Final Report, Department of Defense CounterDrug Technology Office, 1997.

[11] C. Burger, Design of a Roll Control System for a Sailplane Using All-Wing Deflections with Adaptive Materials, Auburn University, Birmingham, Ala, USA, 2001.

[12] "Dynalloy Mechanical Properties," December 2004, http:// www.dynalloy.com/.

[13] D. Stöckel, in The Martensitic Transformation in Science and Technology, E. Hornbogen and N. Jost, Eds., p. 223, Informationsgesellschaft, 1989.

[14] D. Padgett, The Design and Construction of a Shape Memory Alloy Tail Boom Actuator, Auburn University, Birmingham, AL, USA, 2002. 

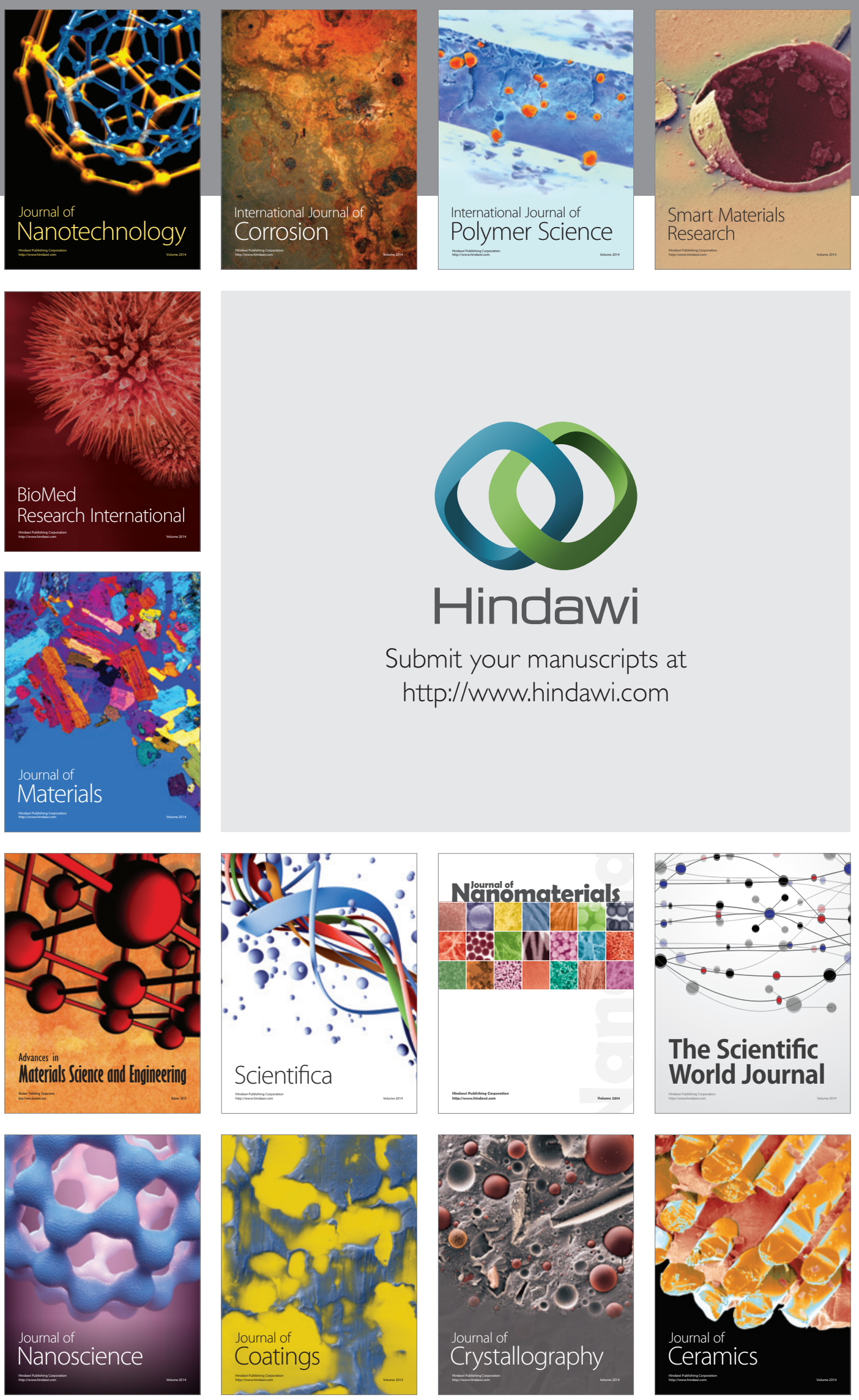

The Scientific World Journal

Submit your manuscripts at

http://www.hindawi.com

\section{World Journal}

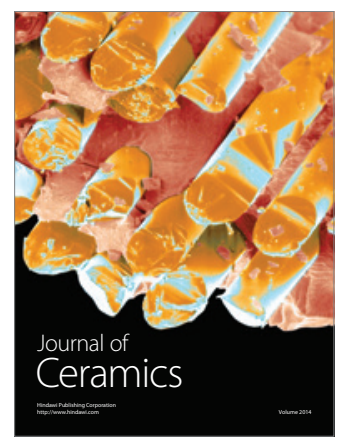

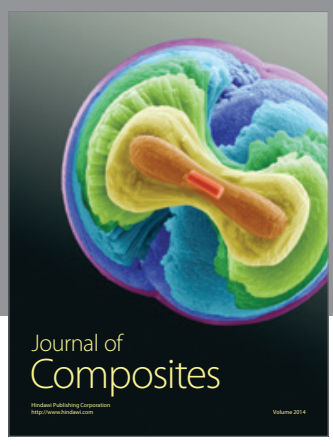
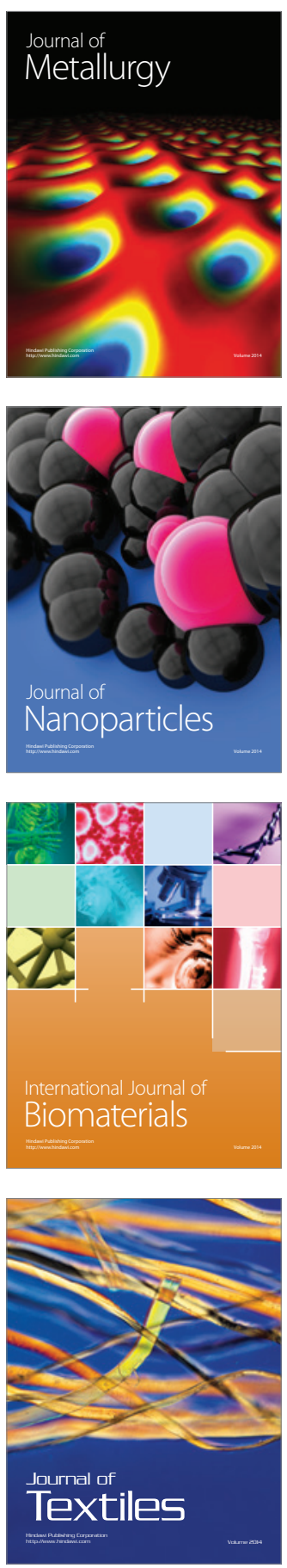